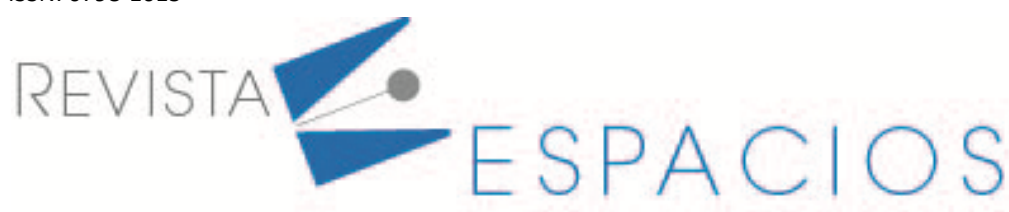

Vol. 42 (10) $2021 \cdot$ Art. 2

\title{
Participación de la Corporación del Seguro de Depósitos, Fondo de Liquidez y Fondo de Seguros Privados (COSEDE) en el sistema financiero ecuatoriano. Período 2013 - 2018
}

\author{
Participation of the deposit insurance corporation, liquidity fund and private insurance fund \\ (COSEDE) in the ecuadorian financial system. Period 2013- 2018
}

\author{
PLUAS, Vanessa A. ${ }^{1}$ \\ GARABIZA, Bella R. ${ }^{2}$
}

\begin{abstract}
Resumen
La Corporación del Seguro de Depósitos, Fondo de Liquidez y Fondo de Seguros Privados (COSEDE) en el sistema financiero ecuatoriano durante el período 2013 - 2018 ha presentado un constante crecimiento de los fondos que gestiona, como el fondo del seguro de depósitos en el Sector Financiero Privado y el Sector Financiero Popular y Solidario que busca resguardar los ahorros de la población ecuatoriana, entre otros. La metodología aplicada fue cualitativa y hubo una revisión documental, para obtener información de este organismo, los mecanismos que administra y su evolución, y los servicios que brinda.

Palabras clave: sistema financiero ecuatoriano, proceso de liquidación, fondo de liquidez, fondo del seguro de depósitos.
\end{abstract}

\begin{abstract}
The Deposit Insurance Corporation, Liquidity Fund and Private Insurance Fund (COSEDE) in the Ecuadorian financial system during the period 2013 - 2018 has presented a constant growth of the funds that it manages as: the deposit insurance fund in the Sector Private Finance and the Popular and Solidarity Financial Sector that seeks to protect the savings of the Ecuadorian population, among others. The applied methodology was qualitative and there was a documentary review to obtain information on this body, the mechanisms it manages and its evolution, and the services it provides.

Key words: ecuadorian financial system, liquidation process, liquidity fund, deposit insurance fund.
\end{abstract}

\section{Introducción}

A partir de la expedición de la Ley de Creación de la Red de Seguridad Financiera se estipuló la creación de la Corporación del Seguros de Depósitos, Fondos de Liquidez y Fondo de Seguros Privados (COSEDE), cuyo propósito será el de administrar dos mecanismos de la red: el fondo de liquidez y el sistema de seguro de depósitos, para inspeccionar y controlar el sistema financiero ecuatoriano.

El fondo de liquidez será el encargado de cubrir las deficiencias de liquidez y solvencia de las instituciones del Sector Financiero Privado (SFP) y el Sector Financiero Popular y Solidario (SFPS) que presente problemas en su

\footnotetext{
${ }^{1}$ Economista de la Universidad de Guayaquil, vanessa.pluasz@ug.edu.ec

${ }^{2}$ Economista y Magíster en Finanzas y Proyectos Corporativos. Docente de la Facultad de Ciencias Económicas de la Universidad de Guayaquil, Doctorando en Ciencias Económicas - Mención Administración. Universidad Nacional de Cuyo de Argentina, bella.garabizac@ug.edu.ec
} 
patrimonio, es decir actúa como prestamista de última instancia. Mientras que el fondo de seguro de depósitos actuará cuando una entidad financiera se encuentre en liquidación forzosa, para proteger los ahorros de los ciudadanos a través del monto de cobertura que ofrece a cada entidad financiera.

Entre las funciones que tiene este organismo esta la de brindar información a la ciudadanía acerca de las gestiones y servicios que ofrecen. El objetivo fundamental del presente trabajo de investigación es analizar la participación de la Corporación del Seguros de Depósitos, Fondos de Liquidez y Fondo de Seguros Privados (COSEDE) en el sistema financiero ecuatoriano, período 2013 - 2018. Mediante la aplicación de las encuestas se pretende determinar los niveles de desconocimiento que tiene actualmente la población ecuatoriana sobre la existencia de esta institución.

\subsection{Asociación Internacional de Aseguradores de Depósitos (IADI)}

En mayo de 2002 se fundó la Asociación Internacional de Aseguradores de Depósitos (IADI, por sus siglas en inglés), con el objetivo de mejorar la seguridad de los sistemas de seguro de depósitos a través de promover una adecuada disposición y cooperación internacional. Esta institución ofrece todo tipo de programas de educación, investigación y consejos acerca de los seguros de depósitos, para los países que quieren implementar o perfeccionar el sistema de seguro de depósitos, mediante las conferencias internacionales y foros que realizan. La Asociación Internacional de Aseguradores de Depósitos (IADI) actualmente cuenta con 91 países miembros, 10 asociados y 14 socios.

Un sistema bancario sólido y competitivo es importante para la vitalidad económica de una nación. Los bancos prestan servicios críticos a través de su función en el sistema de pagos, en la intermediación de fondos de los ahorradores a los consumidores e inversores, y en la transmisión de la política monetaria. Un elemento clave para mantener la confianza en el sistema bancario y promover la estabilidad financiera es el seguro de depósitos. El número de jurisdicciones que establecen o consideran establecer un sistema de seguro de depósitos se ha expandido rápidamente en los últimos años. (Asociación Internacional de Aseguradores de Depósitos, n.d)

\subsection{El Comité de Supervisión Bancaria de Basilea (BCBS)}

EI BCBS ha sido desde su formación ha sido el organismo normativo internacional para la regulación prudencial de los bancos y constituye un foro de cooperación continua en materia de supervisión bancaria, su mandato es mejorar la regulación, supervisión, prácticas bancarias y gestión de riesgos a nivel mundial con el fin de lograr la estabilidad financiera. (Rivas García, 2016, pág. 7)

El comité elabora las normas globales de control, realiza la emisión de reglas y recomienda habilidades, para que las autoridades de control de todos los países las apliquen a sus sistemas financieros nacionales según las peculiaridades y sistema bancarios locales para conocer de qué manera se adaptaran las prácticas propuestas.

\subsubsection{Principios básicos para Sistemas de Seguro de Depósitos eficaces según el Comité de Supervisión Bancaria de Basilea}

El Comité de Supervisión Bancaria de Basilea en conjunto con la Asociación Internacional de Aseguradores de Depósitos (IADI), en 2009 estableció los principios básicos para un sistema de seguro de depósitos eficaces. En 2010, se perfeccionó la metodología de los principios básicos.

Los principios básicos han cumplido adecuadamente su propósito de ayudar a las jurisdicciones a evaluar sus sistemas de seguro de depósitos e identificar áreas de mejora. Tanto los principios básicos como su metodología de evaluación se han recopilado en un solo documento, haciendo cuidadosos esfuerzos por mantener la continuidad y la posibilidad de comparación (Asociación Internacional de Aseguradores de Depósitos, 2016, pág. 6). Los cuales se mencionarán a continuación:

Principio 1. Objetivos de la política pública: Los objetivos fundamentales que se deben considerar dentro de esta política es la protección de los depositantes y la asistencia de la estabilidad financiera. 
Principio 2. Mandato y poderes: El mandato y los poderes del asegurador o la institución deberán estar rotundamente definidos para responder a las finalidades de la política pública.

> Principio 3. Gobernanza: Se recomienda que la institución o el asegurador del depósito ser independiente, transparente, responsable y no tenga influencias externas.

Principio 4. Relación con otros participantes de la red de seguridad financiera: Para cumplir con los objetivos de la política pública tiene que existir un marco legal regulatorio, hacia la ejecución de las actividades y la reciprocidad de la información entre el asegurador y otras entidades de la red de seguridad financiera.

Principio 5. Temas transfronterizos: Los bancos extranjeros se comprometen a intercambiar la información acerca de los convenios formales para que haya una coordinación de los seguros de depósitos entre la matriz y las sucursales de los aseguradores de depósitos.

$>$ Principio 6. El papel del seguro de depósitos en la planificación de contingencias y manejo de crisis: La institución aseguradora de depósitos tiene que elaborar planes de contingencia, como políticas e instrucciones para hacer frente a crisis, para asegurar una respuesta inmediata ante una emergencia bancaria u otros. La elaboración de las políticas e instrucciones es responsabilidad de todos los participantes de la red de seguridad financiera.

$>$ Principio 7. Membresía: La membresía del sistema de seguros de depósitos es un compromiso entre todas las instituciones bancarias.

> Principio 8. Cobertura: Cuando se elaboren las políticas e instrucciones acerca del seguro de depósitos se tiene que precisar los niveles y alcance de la cobertura de este. La cobertura puede ser restringida, fidedigna y proteger a los depositantes ya que esta debe responder a los objetivos de las políticas públicas referidos al sistema de seguros de depósitos.

$>$ Principio 9. Origen y usos de los fondos: La institución aseguradora tiene que poseer de disponibilidad inmediata de los fondos y tener los componentes de financiamiento precisos para aseverar el pago de los ahorros a los depositantes. Los responsables del pago del seguro de depósitos son las entidades bancarias.

$>$ Principio 10. Concientización del público: Con el objetivo de resguardar los ahorros de los clientes y favoreces a la estabilidad financiera, es necesario que se realicen difusión constantemente al público en general acerca de los beneficios y restricciones del sistema de seguro de depósitos.

$>$ Principio 11. Protección legal: Los integrantes que laboran en la institución aseguradora de los depósitos tienen que estar capacitados para las acciones del público como: reclamos, demanda entre otros ejercicios.

$>$ Principio 12. Manejo de los responsables de una quiebra bancaria: La autoridad principal de la entidad tiene que poseer la potestad de averiguar compensaciones legales por parte de los responsables de la quiebra de una institución bancaria.

$>$ Principio 13. Detección temprano e intervención oportuna: El asegurador debe ser parte del esquema de la red de seguridad financiera, la cual ayuda a detectar anticipadamente los problemas bancarios para que haya la intervención necesaria hacia su corrección. Entre las acciones que tienen que aplicarse, está la protección de los ahorros de los depositantes.

$>$ Principio 14. Resolución: Una fuerte resolución permitirá a la autoridad principal proponer el resguardo de los depósitos y la estabilidad financiera.

Principio 15. Reembolso a depositantes: El fondo de seguro de depósitos devolverá los ahorros de manera oportuna a los clientes para lograr la estabilidad financiera. Deben tener una planificación para el pago a los depositantes afianzados. 
Principio 16. Recuperación de activos: La institución aseguradora tiene, por ley, el derecho a rescatar los créditos de los acreedores determinados.

\subsection{Antecedentes de la investigación}

Los seguros de depósitos surgieron como una medida de prevención de pérdida de patrimonio luego de sucesos de pánico bancario, perturbaciones financieras provocados por las dificultades bancarias que se han dado a la largo de la historia, finalizando con el desplome de las instituciones financieras. El primer escenario se observó en Checoslovaquia en 1924, luego en Estados Unidos de América en 1933 por la crisis que sufrió llamada la Gran Depresión, siendo desde aquí en donde muchos países tomaron como ejemplo aquellas situaciones para incorporar este mecanismo. Para el 2008 una crisis financiera reventó en Estados Unidos de América debido al excesivo otorgamiento de créditos de la banca privada sin la respectiva evaluación hacia sus clientes, ocasionando que las fuerzas de mercado (oferta y demanda) encontraran su equilibrio en bajos niveles de precios ocasionando pérdidas millonarias.

En Ecuador la crisis financiera “inició en 1994 donde las instituciones financieras ofrecían grandes tasas de interés que llegaron al $270 \%$, con el fin de viabilizar los ahorros de las empresas extranjeras y de los pequeños agricultores, artesanos y clientes en general, quienes buscaban resguardar las instituciones financieras, con una apariencia de negocio parecía rentable." (Apolinario Quintana, Valle, \& Caicedo , 2017)

La situación del país desmejoró a finales de los años 90 por una serie de factores como el fenómeno de El Niño en 1998, los bajos precios del petróleo que iniciaron en 1998 y se prolongaron hasta 1999, la deuda externa, entre otros. Fue aquí en donde el Estado asumió la crisis, pero se empezaron a utilizar el dinero de todos los ecuatorianos para reparar el sistema financiero. En 1999 la economia ecuatoriana colapsó y el presidente de ese año, Jamil Mahuad Witt, decidió eliminar la moneda nacional llamada sucre por una moneda internacional, el dólar americano. Esta medida generó efectos socioeconómicos negativos como: la migración, empobrecimiento, suicidios, abandono del grupo familiar, destrucción de la confianza en el sistema financiero ecuatoriano.

El sistema financiero internacional había colapsado aquel año, y aquellos países e instituciones financieras con deudas no pudieron recuperar el dinero invertido por haber invertido en compras de hipotecas que fueron calificadas como "ninjas", es decir, deudas que pertenecían a clientes sin ingresos, sin empleo y sin activos. (Garabiza, 2014, pág. 29).

En el año 2008, la Asamblea Constituyente aprobó la normativa en la que se preinscribió “la Ley de Creación de la Red de Seguridad Financiera, publicada en el tercer suplemento del Registro Oficial No. 398 del 31 de diciembre de 2008; Ley en la cual, por primera vez en la historia del Ecuador, se daba un ordenamiento coordinado de las instituciones públicas llamadas a precautelar la estabilidad financiera del país, en una red donde las responsabilidades se definían y se asignaban de acuerdo con la naturaleza técnica de cada institución, pero siempre bajo el espíritu de un trabajo coordinado en perfecto complemento de objetivos interinstitucionales" (Corporación del Seguro de Depósitos Fondo de Liquidez y Fondo de Seguros Privados, 2014)

\subsection{La Red de Seguridad Financiera en Ecuador}

La Red de Seguridad Financiera es parte importante de la arquitectura del sistema financiero de un país que requiere, además, un ordenamiento jurídico transparente, instituciones de supervisión y control efectivas, un entorno macroeconómico adecuado y seguro y sólidas instituciones financieras (Guerrero, Focke, \& Rossini, 2010, pág. 5)

Estas redes tienen como objetivo principal buscar o elaborar los mecanismos necesarios para reducir los riesgos de las futuras quiebras de las entidades bancarias debido a las crisis financieras, evitando así que sean pocas las instituciones que colapsen a la vez, siendo esta la manera más eficaz de proteger el sistema financiero de un país. 
El 31 de diciembre de 2008, se estableció la Ley de Creación de la Red de Seguridad Financiera publicada en el Registro Oficial No. 498, en donde la nueva arquitectura financiera ecuatoriana busca la creación de una Red de Seguridad Financiera, la cual está integrada por cuatro mecanismos que se mostrarán en la figura 1.

Figura 1

Mecanismo de la Red de Seguridad Financiera Ecuatoriana

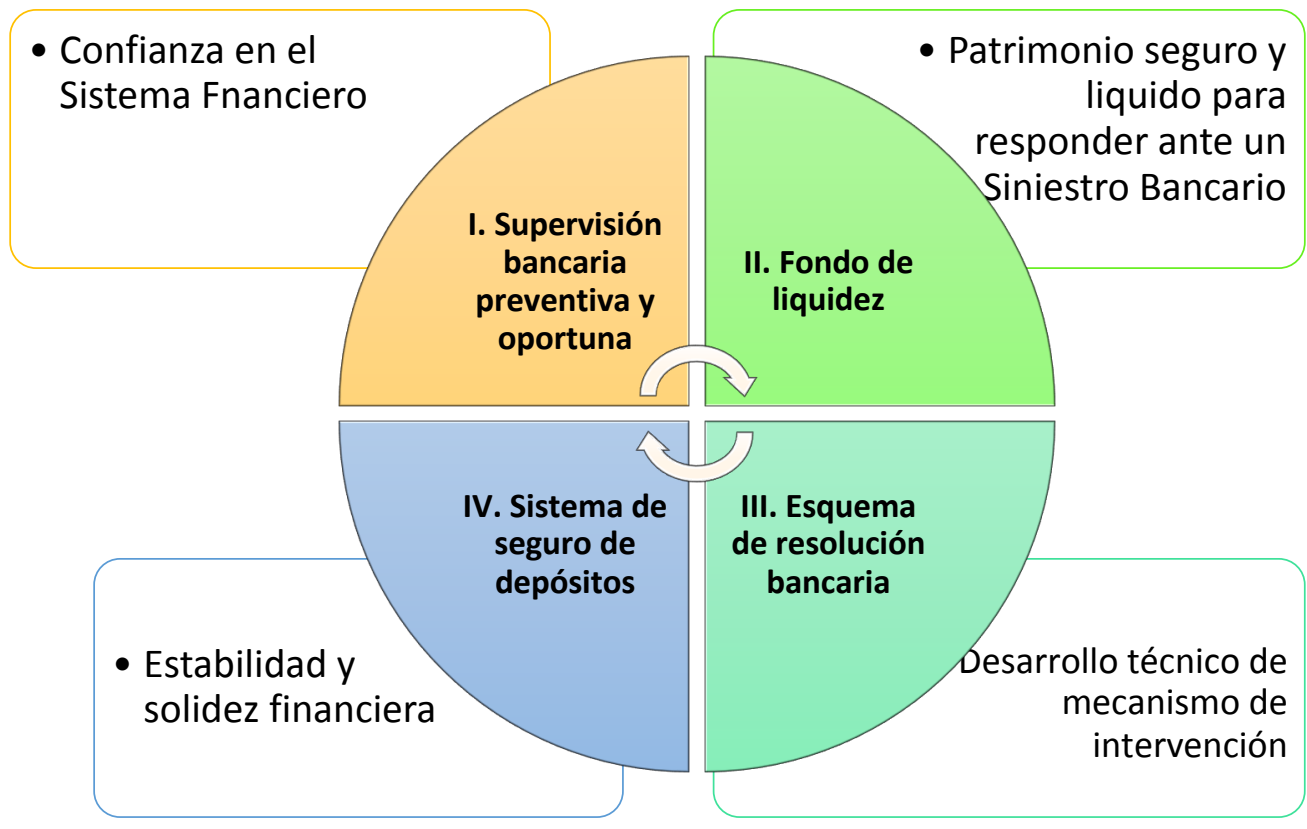

Fuente: Corporación del Seguro de Depósitos, Fondo de Liquidez y Fondo de Seguros Privados. Elaborado por las autoras.

\subsubsection{Supervisión bancaria preventiva y oportuna}

La entidad que está encargada de este mecanismo es la Superintendencia de Bancos (SB), la cual tiene como objetivo minimizar los riesgos que puede producir la liquidación forzosa de una institución financiera. Para esto, se debe asumir un sistema de supervisión continua y de carácter correctivo con el propósito de pulir los niveles de eficiencia en dichas entidades, además, la ejecución de campañas de información y educación para acrecentar los niveles de cultura financiera en la ciudadanía.

\subsubsection{Fondo de liquidez}

Este fondo no percibe recursos públicos, está conformado por las contribuciones de las instituciones financieras, rendimientos de inversiones, donaciones y otros ingresos como prestamos, líneas contingentes, según este estipulado en la Ley. El organismo que lo controla y administra es el Banco Central del Ecuador (BCE), quien opera como prestamista de última instancia a las IFIS ante la presencia de falta de liquidez.

\subsubsection{Esquema de resolución bancaria}

La Junta de Política y Regulación Monetaria y Financiera (JPRMF) a través de la Ley de Creación de la Red de Seguridad Financiera, es la entidad encargada de desarrollar todos los procedimientos y medidas que buscan mejorar el panorama de una liquidación forzosa de una institución financiera procurando salvaguardar los depósitos de los ciudadanos.

Para precautelar los depósitos de los ciudadanos, la Superintendencia de Bancos (SB) y la Junta de Política y Regulación Monetaria y Financiera (JPRMF) dispondrán de la suspensión de toda operación bancaria para las transferencias de activos y pasivos de una entidad financiera a otra con el fin de que sean administradas 
eficientemente, luego se asignará un Administrador Temporal o Liquidador, quien será el encargado de elaborar los informes como: el balance del proceso ETAP, el balance residual y el aporte de la COSEDE.

\subsubsection{Sistema de seguro de depósitos}

Cuando la Junta de Política y Regulación Monetaria y Financiera (JPRMF) comunique el pago de los depósitos, la Corporación de Seguros de Depósitos, Fondo de Liquidez y Fondo de Seguros Privados (COSEDE), entidad comisionada de la devolución, se encargará de elaborar un calendario para la respectiva restitución según el monto de cobertura que posea la institución financiera

\subsection{La Corporación del Seguro de Depósitos, Fondo de Liquidez y Fondo de Seguros Privados (COSEDE)}

En el artículo 13 de la Ley de Creación de la Red de Seguridad Financiera, se estipula la creación de la Corporación de Seguro de Depósitos, Fondo de Liquidez y Fondo de Seguro Privados (COSEDE), institución de carácter público, pero con independencia administrativa y operativa. Teniendo como objetivo la gestión del sistema de seguro de depósitos, la cual se establecerá a partir de las aportaciones que realice la institución financiera.

La COSEDE responderá principalmente a los depositantes ante la presencia de una liquidación forzosa o cierre de una agencia del Sistema Financiero Privado, siempre y cuando dicha entidad se encuentre respaldada por el seguro.

En el 2011 bajo el registro oficial № 444, entró en vigor la Ley Orgánica de Economía Popular y Solidaria del Sistema Financiero, en el que se encuentra la incorporación de un fondo de liquidez y de seguro de depósitos, para proteger todos los ahorros de las Cooperativas de Ahorro y Créditos (COAC), entidades asociativas y solidarias, cajas y bancos comunales y cajas de ahorros regularizados por la ley.

\subsubsection{Mecanismos de la Corporación del Seguro de Depósitos, Fondo de Liquidez y Fondo de Seguros Privados (COSEDE)}

En el año 2014 con la creación del Código Orgánico Monetario y Financiero, se integró a la COSEDE nuevas competencias, convirtiéndola en la administradora de tres mecanismos financieros, como los son: el fondo de seguro de depósitos, el fondo de liquidez y el fondo de seguros privados. Los cuales se detallarán a continuación:

1.5.1.1. Fondo de seguros de depósitos. Es un mecanismo de contingencia financiera que tiene como finalidad resguardar los depósitos de todos los clientes de las entidades financieras veladas por el fondo, cuando se presente una liquidación forzosa.

1.5.1.1.1. Composición del seguro de depósito. Según la Ley de Creación de la Red de Seguridad Financiera, el seguro de depósitos se encuentra compuesto por los siguientes rubros:

$>$ El aporte inicial del Fondo Especial de la Agencia de Garantía de Depósitos (AGD);

DEl aporte que efectuarán las instituciones del sistema financiero (IFIS);

$>$ El beneficio de las inversiones y las utilidades líquidas de cada ejercicio anual del Fondo de Seguro de Depósitos;

$>$ Las donaciones que percibe la Corporación de Seguros de Depósitos, Fondo de Liquidez y Fondo de Seguros Privados; $y$,

$>$ Los préstamos o líneas de contingentes, las cuales son destinadas para el financiamiento de sus actividades.

1.5.1.1.2. Monto de cobertura. En la figura 2 se presenta la clasificación de la cobertura del seguro de depósitos por sector financiero: 


\section{Figura 2}

Monto de cobertura del fondo de seguro de depósitos

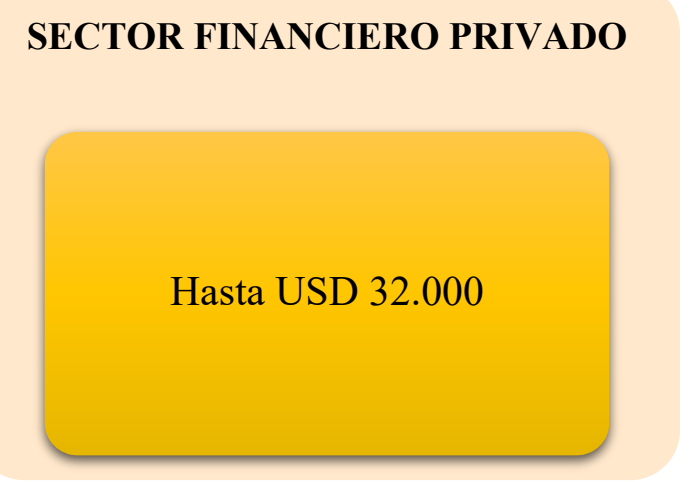

\section{SECTOR FINANCIERO POPULAR Y SOLIDARIO}

Segmento 1: Hasta USD 32.000

Segmento 2: Hasta USD 11.290

Segmento 3: Hasta USD 5.000

Segmento 4: Hasta USD 1.000

Segmento 5: Hasta USD 1.000

Fuente: Corporación del Seguro de Depósitos, Fondo de Liquidez

y Fondo de Seguros Privados. Elaborado por las autoras.

1.5.1.2. Fondo de liquidez. Tiene como objetivo atender las necesidades de liquidez y solvencia que presenten las instituciones del Sector Financiero Privado y del Sector Financiero Popular y Solidario, a través de dos fideicomisos independiente para conservar el patrimonio técnico dentro de los niveles exigidos por la ley.

Este fondo realiza operaciones activas como créditos cuyo plazo será de un día hábil renovable, dentro de una línea de crédito, para cubrir deficiencias de las entidades financieras en las cámaras de compensación del Sistema Nacional de Pagos administrado por el Banco Central del Ecuador, créditos para solucionar necesidades extraordinarias de liquidez de las entidades financieras privadas y del Sector Financiero Popular y Solidario; cuyo plazo máximo será de hasta 365 días a partir de su concesión, además de otros préstamos de liquidez que pueda aprobar la Junta de Política y Regulación Monetaria y Financiera (Corporación del Seguro de Dépositos, Fondo de Liquidez y Fondo de Seguros Privados, n.d).

1.5.1.3. Fondo de seguros privados. Es el encargado de proteger a las personas naturales y jurídicas tanto públicas como privadas que tengan pólizas vigentes en las aseguradoras del Sistema de Seguros Privados, en el caso que una institución de este sistema sea notificada en liquidación forzosa.

Este seguro funciona a través de un fideicomiso mercantil constituido en el Banco Central del Ecuador y presenta una cobertura, a favor de cada asegurado o beneficiario, por un monto máximo de USD 1.500,00 tomando en cuenta cada siniestro pendiente de pago a la fecha de liquidación forzosa de la empresa de seguros. El fideicomiso se nutre principalmente de los recursos que contribuyen las empresas del sistema de seguros privados y la proporción establecida en el artículo 67 de la Ley General de Seguros. Estos recursos son inembargables y no son sujetos de ninguna medida cautelar (Corporación del Seguro de Dépositos, Fondo de Liquidez y Fondo de Seguros Privados, n.d).

\section{Metodología}

Los aspectos metodológicos que se abordaron en el tema de estudio fue el enfoque cualitativo, mismo que permitió evidenciar la premisa de investigación por medio de la recolección de datos estadísticos acerca del fondo de liquidez, el cual cubrirá el seguro de depósitos, a través de la información que brinda la Corporación de Seguros de Depósitos, Fondos de Liquidez y Fondos de Seguros Privados (COSEDE). Después de la recolección y análisis de datos, el cualitativo permitió analizar la participación que ha tenido la COSEDE en el sistema financiero ecuatoriano durante el período 2013 - 2018.

Este trabajo de investigación utilizó un diseño exploratorio, pues tal como mencionan Hernández Sampieri et al (2014) este enfoque se utiliza cuando el objetivo es examinar un tema poco estudiado o novedoso. 


\subsection{Tipos de investigación}

Tuvo un alcance exploratorio y descriptivo, como el problema de investigación no ha sido estudiado antes, el alcance exploratorio favoreció para conocer los antecedentes de los seguros de depósitos. Por consiguiente, el descriptivo permitió detallar las propiedades de la COSEDE, la composición y características de los seguros de depósitos.

\subsection{Técnicas e instrumentos de la investigación}

La investigación documental a través de sus técnicas e instrumentos permitió conseguir información relevante que posibilitó conocer puntos determinados sobre la situación a estudiar, en este caso utilizando las fuentes primarias y secundarias de investigación, las cuales a través de revisión bibliográfica brindan un panorama más amplio sobre el conocimiento de la población en temas financieros y facilita el estudio de esta.

El instrumento que se utilizó en la investigación de campo son las encuestas escrita, ya que estas ayudaron a obtener referencia acerca del conocimiento que tiene la población en general acerca la Corporación de Seguro de Depósitos, Fondo de Liquidez y Fondo de Seguros Privados (COSEDE) y la participación que ha tenido en las liquidaciones de entidades financieras nacionales.

Las encuestas fueron dirigidas a todas las personas que poseen una cuenta de ahorro en las diferentes instituciones financieras y para la aplicación de la misma se movilizó a las afueras de las entidades bancarias a conocer la opinión de cada ciudadano y a través de formulario de Google para llegar a más habitantes del país.

\subsection{Población y muestra}

\subsubsection{Población}

Según el Instituto Nacional de Estadísticas y Censo (INEC), la proyección de la población, según grupo de edad período 2010 - 2020 para el año 2018, fue de 17.023 .408 personas, de las cuales se seleccionó el rango de edad comprendido de 15 hasta 49 años, información que fue escogida para obtener la población de estudio (ver anexo 2).

\subsubsection{Muestra}

Esta se caracteriza por ser una parte representativa de la población ya que es más accesible su estudio. Se utilizó la fórmula para la elección del tamaño de la muestra de una población finita con carácter probabilístico, la cual es:

$$
n=\frac{(1,96)^{2}(0,50 * 0,50) 8^{\prime} 753.202}{(1,96)^{2}(0,50 * 0,50)+\left(8^{\prime} 753.202-1\right)(0,05)^{2}}=\mathbf{3 8 4}
$$

Donde:

$\mathrm{n}$ : tamaño de la muestra, fue de 384.

$\mathrm{N}$ : tamaño de la población, que es de 8.753.202.

p: posibilidad de que suceda un evento, $p=0,50$.

q: posibilidad de que no suceda un evento, $q=0,50$.

e: error, e = 0,05; el cual representa el $5 \%$.

z: nivel de confianza, el cual se escogió el $95 \%$, dando como valor z $=1,96$.

\section{Resultados}

\subsection{Evolución del fondo de liquidez}

Este fondo es el encargado de responder ante la falta de liquidez de las instituciones del Sector Financiero Privado y del Sector Financiero Popular y Solidario, a través de créditos o préstamos. A continuación, se presenta el fondo para cada sector financiero: 
Cuadro 1

Proyección de población, según grupo

de edad. Año 2018 (en millones USD)

\begin{tabular}{cccc}
\hline Años & Fondo de liquidez SFP & Fondo de liquidez SFPS & Fondo de liquidez consolidado \\
\hline 2013 & $1.642 .745 .777,60$ & - & $1.642 .745 .777,60$ \\
2014 & $2.114 .927 .889,22$ & - & $2.114 .927 .889,22$ \\
2015 & $2.233 .255 .684,69$ & - & $2.233 .255 .684,69$ \\
2016 & $2.390 .884 .246,13$ & $66.681 .805,32$ & $2.457 .566 .051,45$ \\
2017 & $2.517 .219 .136,22$ & $107.916 .485,74$ & $2.625 .135 .621,96$ \\
2018 & $2.645 .825 .627,12$ & $161.779 .590,33$ & $2.807 .605 .217,45$ \\
Total & $13.544 .858 .360,98$ & 336.377.881,39 & $13.881 .236 .242,37$ \\
\hline \multicolumn{4}{c}{ Fuente: Corporación del Seguro de Depósitos, Fondo de } \\
Liquidez y Fondos de Seguros Privados (COSEDE). \\
\multicolumn{4}{c}{ Elaborado por las autoras. }
\end{tabular}

En el cuadro 1, se detallan los valores que se recaudan anualmente en el fondo de liquidez del sector financiero privado, sumando un total de USD 13.544.858.360 los cuales ayudaron a que el organismo brindará liquidez y solvencia a las instituciones que estaban atravesando problemas de patrimonio, ya que las entidades financieras del sector privado deben mantener un patrimonio técnico no menor al $9 \%$. Mientras que el sector financiero popular y solidario empezó a acumular reservas en el presente fondo desde el año 2016, hasta la actualidad cuenta con un valor de USD 336.377.881.

\subsection{Evolución del fondo de seguros privados}

Para diciembre de 2015 se firmó el contrato del fondo de seguros privados, con la finalidad que desde enero del 2016 el país resguarde los depósitos del Sistema de Seguros Privados, conforme lo estipula el artículo 344 del COMF. El fondo empezó ha registrase a partir del año 2017 y su crecimiento ha sido constante ya que en menos de dos años pasó de USD 1.771 millones en diciembre de 2016 a USD 30.205 millones en diciembre de 2018, teniendo un crecimiento de 28.434 millones de dólares. El fondo de seguros privados posee un valor de USD 48.733.478,72 para hacer frente a liquidaciones forzosas que sucedan en el Sistema de Seguros Privados. A continuación, se mostrará la evaluación del fondo.

\section{Figura 3}

Evolución del fondo de seguros privados

(en millones USD). Período 2013 - 2018

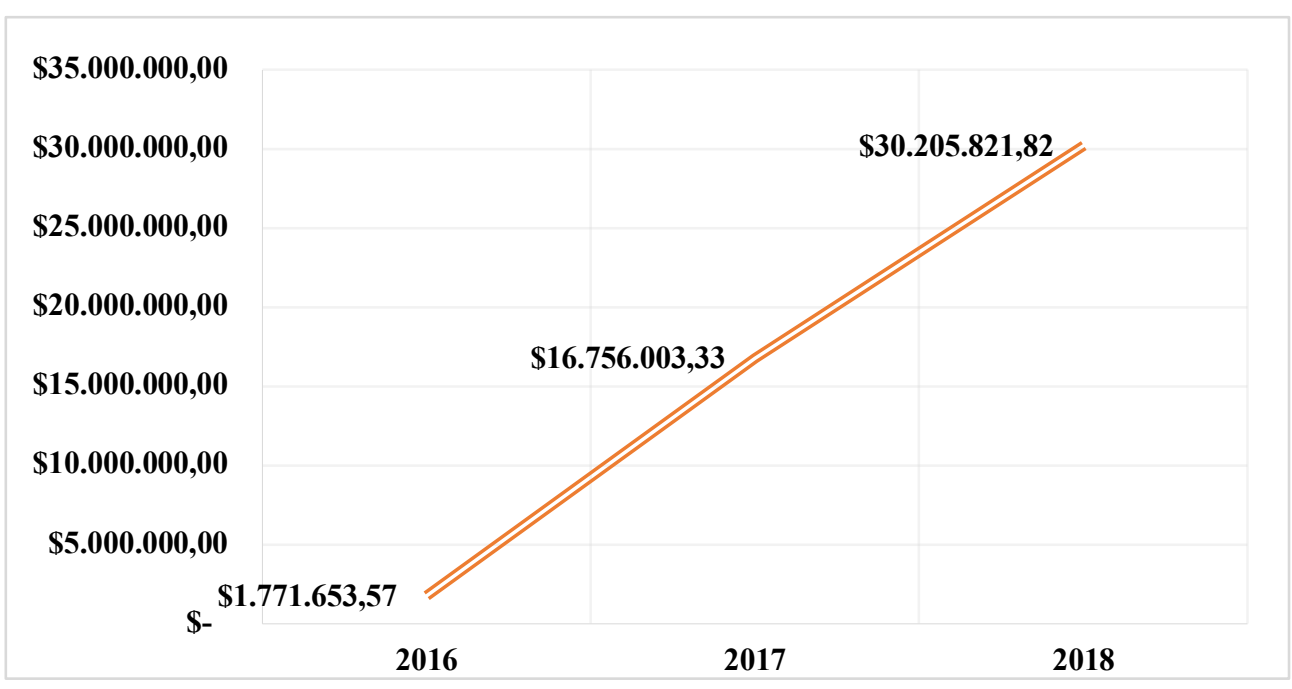

Fuente: Corporación del Seguro de Depósitos, Fondo de Liquidez y Fondos de Seguros Privados (COSEDE). Elaborado por las autoras. 


\subsection{Evolución del fondo de seguro de depósitos}

El presente fondo está conformado por las contribuciones que mensualmente efectúan las entidades financieras del país y por los rendimientos o beneficios que perciben de las inversiones que realiza la COSEDE. Y es destinado netamente a resguardar los depósitos de los ciudadanos.

\section{Cuadro 2}

Evolución del fondo de seguro de depósitos del Sector

Financiero Privado y del Sector Financiero Popular y

Solidario. Período 2013 - 2018 (en millones USD)

\begin{tabular}{cccc}
\hline Años & Fondo de seguro SFP & Fondo de seguro SFPS & Fondo de seguro consolidado \\
\hline 2013 & $721.287 .741,94$ & $77.059 .613,27$ & $798.347 .355,21$ \\
2014 & $902.376 .567,09$ & $106.931 .249,59$ & $1.009 .307 .816,68$ \\
2015 & $1.089 .147 .156,69$ & $146.370 .680,49$ & $1.235 .517 .837,18$ \\
2016 & $1.235 .529 .963,06$ & $216.827 .105,07$ & $1.452 .357 .068,13$ \\
2017 & $1.391 .981 .505,90$ & $286.146 .891,16$ & $1.678 .128 .397,06$ \\
2018 & $1.600 .512 .597,45$ & $337.100 .875,66$ & $1.937 .613 .473,11$ \\
TOTAL & $6.940 .835 .532,13$ & $1.170 .436 .415,24$ & $8.111 .271 .947,37$ \\
\hline
\end{tabular}

Fuente: Corporación del Seguro de Depósitos, Fondo

de Liquidez y Fondos de Seguros Privados (COSEDE).

laborado por las autoras.

En el cuadro 2, se detallan los valores que se recaudan anualmente en el fondo de seguro de depósitos el Sector Financiero Privado y del Sector Financiero Popular y Solidario, los cuales ayudan a que la entidad se encuentre preparada para casuales siniestros que se puedan generar en las instituciones financieras. El SFP cuenta con USD 6.940.835.532,13 para hacer frente a los depósitos de los clientes, mientras que el SFPS posee USD 1.170.436.415,24.

\subsection{COSEDE territorial}

COSEDE territorial tiene como objetivo brindar capacitaciones técnicas mensuales dirigidos a estudiantes, gerentes de entidades financieras, depositantes, público en general de todas las provincias del Ecuador. A continuación, se mostrarán los lugares que fueron visitados por los funcionarios de la institución:

Figura 4

COSEDE territorial

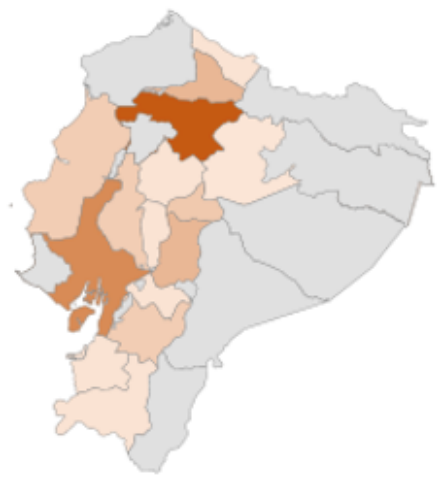

Fuente: Corporación del Seguro de Depósitos, Fondo de Liquidez y Fondos de Seguros Privados (COSEDE). Elaborado por las autoras 
La población ecuatoriana de estudio, según grupo de edad comprendido de 15 hasta 49 años fue de 8.753.202. En la figura 4 se puede observar que de las 24 provincias del Ecuador solamente han sido instruidas 15 de ellas, dando como resultado aproximadamente 2.416 capacitados desde el 2016 hasta el 2018 (ver anexo 1), representando el $0,03 \%$ de la población que saben que son los seguros de depósitos, fondo de liquidez y fondo de seguros privados, los mecanismos de seguridad financiera y el entorno del sistema financiero ecuatoriano.

\subsection{Interpretación de los resultados}

Este epígrafe tiene como objetivo presentar la información adquirida de la aplicación de las encuestas a una muestra estratificada de la población ecuatoriana, que está comprendido en un rango de edad entre 15 hasta 49 años. A continuación, se presenta la interpretación análisis de cada consulta:

El 91\% de la figura 5 describe a los ciudadanos que poseen sus ahorros en alguna institución financiera, mientras que el $9 \%$ decidieron no tomar riesgo y mantener su dinero con ellos, es decir en su casa a través de alcancías, (ver anexo 3).

Figura 5

¿Usted posee sus ahorros en alguna institución financiera del país?

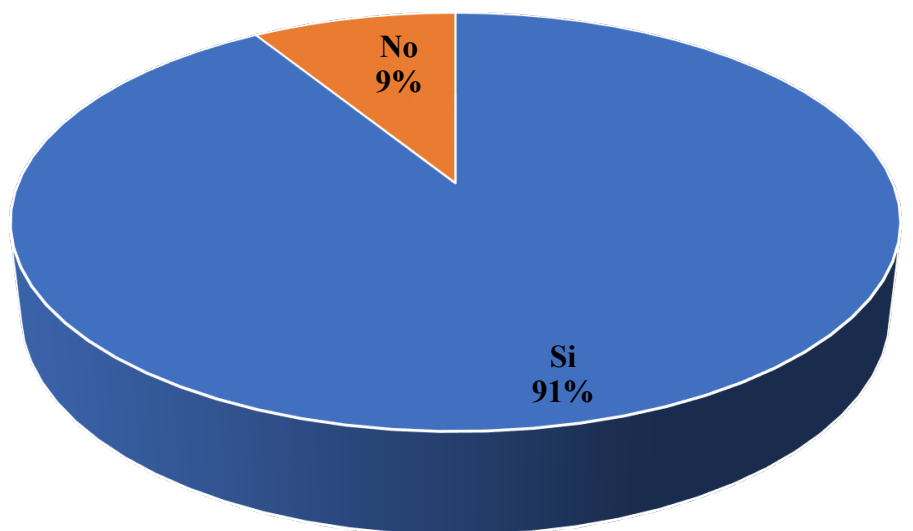

Fuente: Trabajo de campo (encuestas). Elaborado por las autoras.

La cuarta pregunta consistió en conocer en qué tipo de institución financiera los encuestados tienen sus depósitos, obteniendo como resultado que el $78 \%$ de la muestra opto por confiar sus ahorros en las entidades bancarias privadas, el $17 \%$ ahorran en una cooperativa de ahorro y crédito, el $2 \%$ le corresponde a las mutualista y cajas y bancos comunales y el $1 \%$ a las mutualistas. Información que se ve reflejada en la figura 6 (ver anexo 4). 
Figura 6

¿En qué tipo de institución financiera usted tiene sus ahorros?

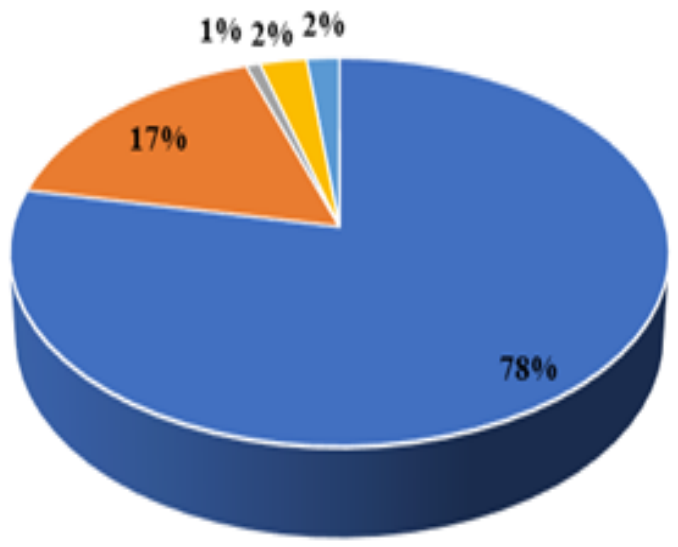

- Entidad Bancaria

- Cooperativa de ahorro y crédito

" Mutualista

Caja y bancos comunales

- Asociaciones

Fuente: Trabajo de campo (encuestas). Elaborado por las autoras.

Con la figura 7 se pudo conocer que el $96 \%$ de la población encuestada se les hace más oportuno mantener su dinero en cuenta de ahorros, la cual no solicita muchos requisitos para su apertura y mantenimiento, el $3 \%$ en cuenta corrientes y el $1 \%$ les corresponde a los depósitos a plazo fijo y obligaciones (ver anexo 5).

\section{Figura 7}

¿En qué tipo de cuenta usted tiene sus ahorros?

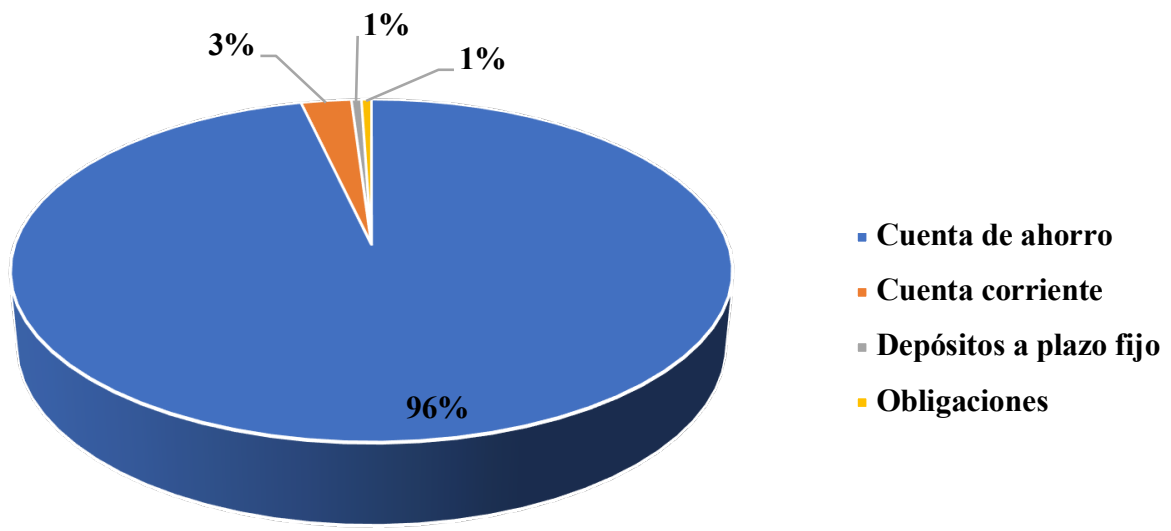

Fuente: Trabajo de campo (encuestas). Elaborado por las autoras.

Con la consulta de saber en qué entidad financiera poseen sus ahorros, se obtuvo que el $47 \%$ de la muestra tiene su dinero en el Banco del Pacífico, el $26 \%$ en el Banco de Guayaquil, el $16 \%$ en el Banco de Pichincha, el $3 \%$ en Produbanco, el $2 \%$ en el Banco Internacional y la Cooperativa JEP, el $1 \%$ corresponden a Banco del Austro Mutualista Pichincha, Banco de Machala y Cooperativa Nacional y el $0 \%$ al Banco Rumiñahui. Información que se ve reflejada en la figura 8 (ver anexo 6). 
Figura 8

Indique en cuál de las siguientes instituciones financieras, usted tiene sus ahorros.

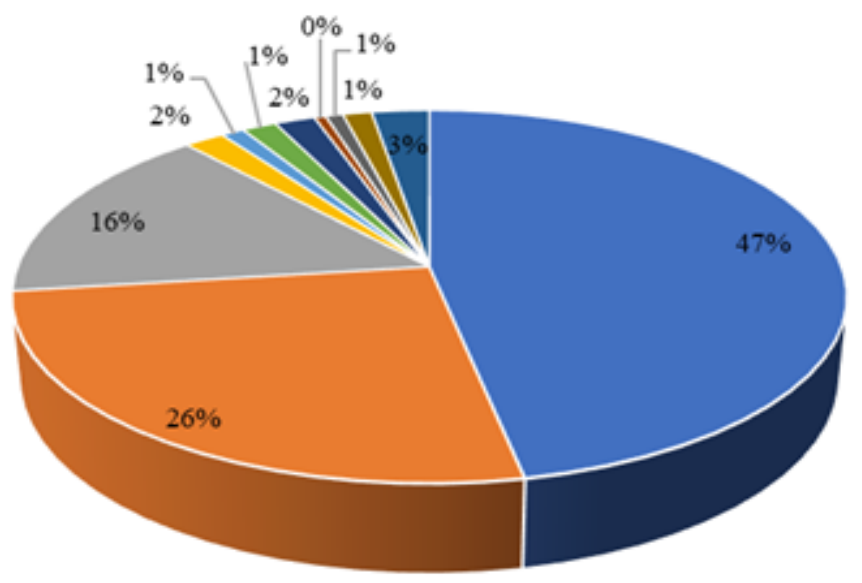

- Banco del Pacifico

= Banco de Guayaquil

- Banco de Pichincha

- Banco Internacional

- Banco de Machala

- Banco del Austro

- Cooperativa Jep

- Banco Rumiñahui

- Mutualista Pichincha

- Cooperativa Nacional

- Produbanco

Fuente: Trabajo de campo (encuestas). Elaborado por las autoras.

En la figura 9 se observa que el $99 \%$ de la muestra reconoce conveniente la decisión de tener una entidad que produzca confianza en el Sistema Financiero, mientras que el 1\% no cree que sea una decisión acertada por acontecimientos pasados (ver anexo 7).

Figura 9

Considera usted acertada la decisión de tener una entidad que genere confianza en el Sistema Financiero y en los depositantes.

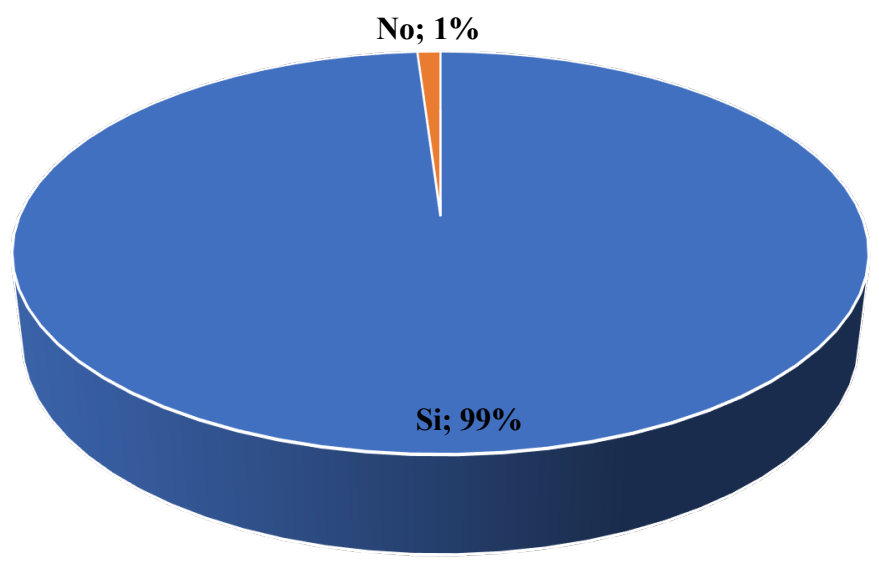

Fuente: Trabajo de campo (encuestas). Elaborado por las autoras.

Se consultó a los encuestados si es que tenían conocimiento acerca que es el seguro de depósitos, consiguiendo como resultado que el $89 \%$ de la muestra no conoce que es este seguro y sus beneficios, mientras que el $11 \%$ posee conocimiento sobre el mismo, información que se visualiza en la figura 10 (ver anexo 8). 
Figura 10

¿Usted conoce que es el

seguro de depósitos?

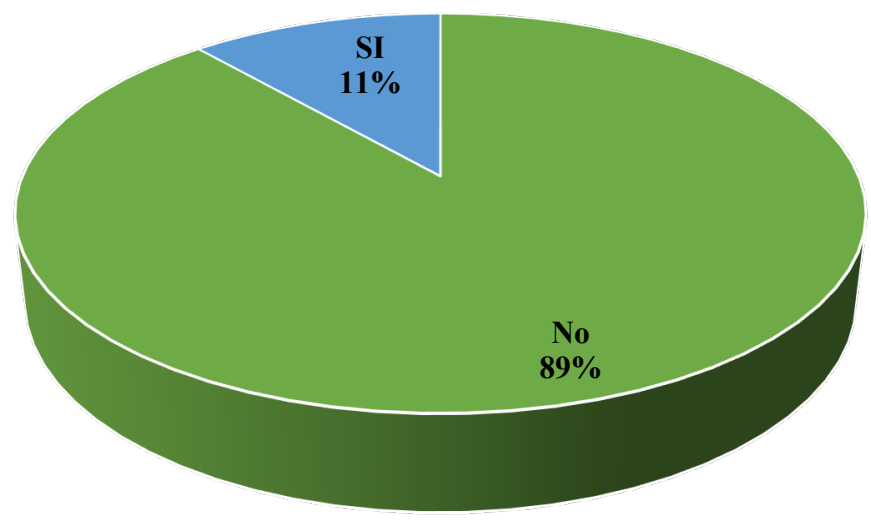

Fuente: Trabajo de campo (encuestas). Elaborado por las autoras.

El 91\% de los encuestados no saben que es la Corporación del Seguro de Depósitos, Fondo de Liquidez y Fondos de Seguros Privado (COSEDE) ni cuáles son sus funciones y gestiones, pero el $9 \%$ dice si conocer acerca de la institución, en la figura 11 se observa los resultados (ver anexo 9).y

Figura 11

¿Usted sabe acerca de la existencia de la Corporación del Seguro de Depósitos, Fondo de Liquidez y Fondos de Seguros Privado (COSEDE)?

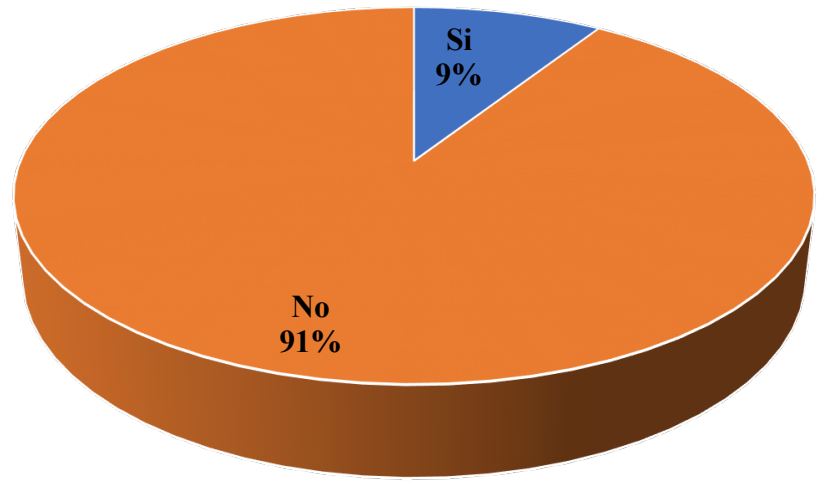

Fuente: Trabajo de campo (encuestas). Elaborado por las autoras.

En la décima pregunta se obtuvo que el $94 \%$ de los encuestados desconocen acerca de las instituciones veladas por la Corporación del Seguro de Depósitos, Fondo de Liquidez y Fondos de Seguros Privado (COSEDE), es decir no saben si el dinero que posee en las diferentes entidades financieras podrán recuperarlo si se llegase a efectuar una liquidación, y el $6 \%$ si conocen sobre las instituciones y es por lo que optaron por depositar sus ahorros allí. Datos que se muestran en la figura 12 (ver anexo 10). 
Figura 12

¿Usted conoce las instituciones veladas por la Corporación del Seguro de Depósitos, Fondo de Liquidez y Fondos de Seguros Privado (COSEDE)?

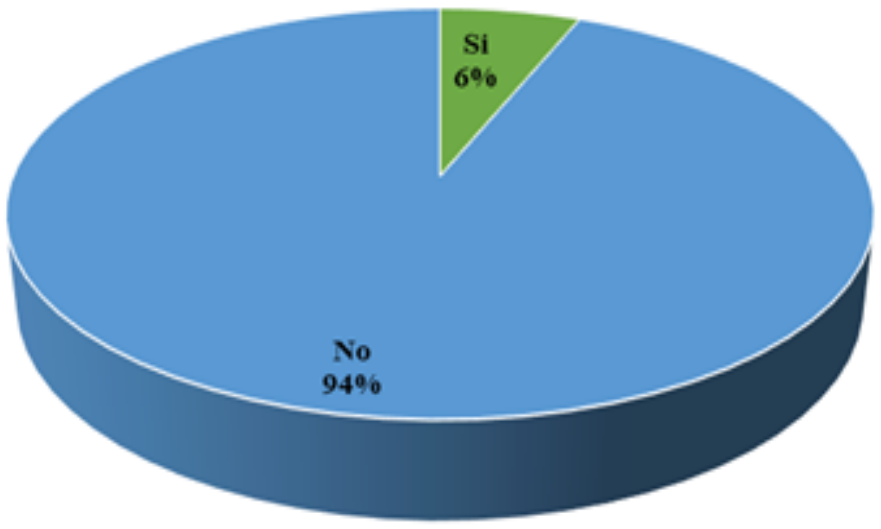

Fuente: Trabajo de campo (encuestas). Elaborado por las autoras.

En la figura 13 se observa que el $95 \%$ de la población encuestada no tiene percepción sobre el monto de cobertura de los depósitos que resguarda la Corporación del Seguro de Depósitos, Fondo de Liquidez y Fondos de Seguros Privado (COSEDE), y el 5\% si conocen cual es el máximo de los ahorros que resguarda la presente institución (ver anexo 11).

\section{Figura 13}

Conoce usted acerca del monto de cobertura de los depósitos que resguarda la Corporación del Seguro de Depósitos, Fondo de Liquidez y Fondos de Seguros Privado (COSEDE).

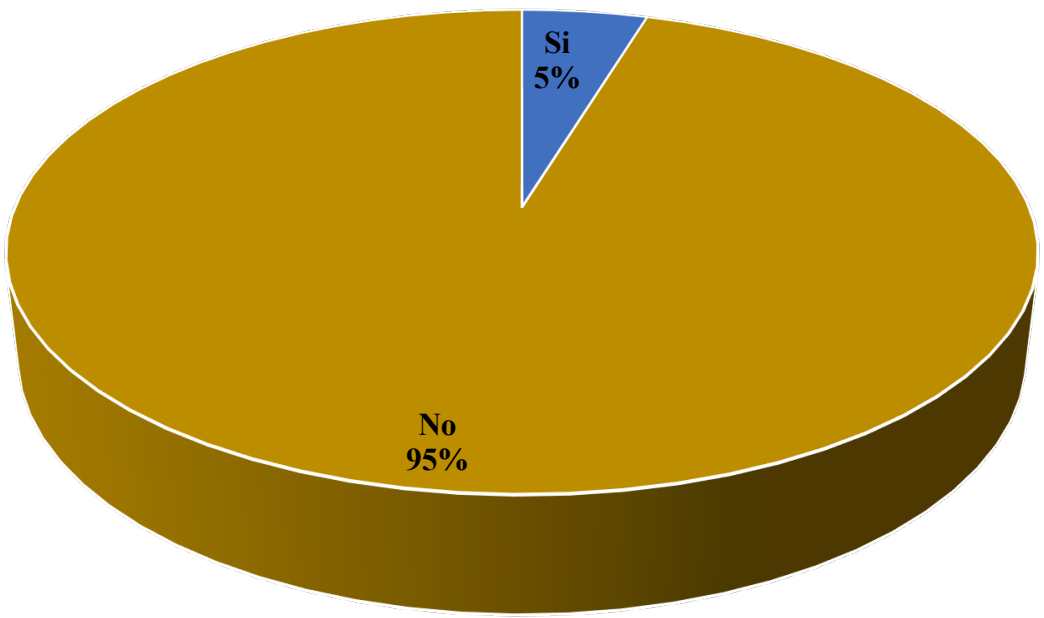

Fuente: Trabajo de campo (encuestas). Elaborado por las autoras.

En la última pregunta del cuestionario se procedió a averiguar si los encuestados se encontraban interesados en obtener más información acerca de las gestiones que realiza la COSEDE y sobre los seguros de depósitos, alcanzando que el $99 \%$ optará por la opción de relacionarse más con el tema financiero, mientras que solo el 1\% prefirió no conocer más, información que se resume en la figura 14 (ver anexo 12). 
Figura 14

Le gustaría recibir charlas informativas acerca de las gestiones que realiza la COSEDE. Adaptado de la información

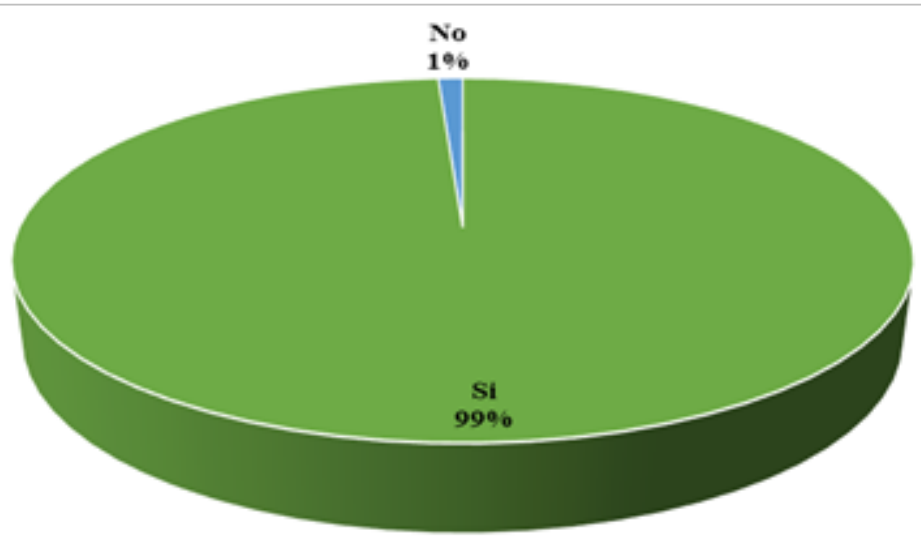

Fuente: Trabajo de campo (encuestas). Elaborado por las autoras.

\subsection{Discusión de los resultados}

La Corporación del Seguro de Depósitos, Fondo de Liquidez y Fondo de Seguros Privados (COSEDE) posee un fondo para asegurar los depósitos del sector financiero privado de USD 6.940.835.532,13, monto que permitirá hacer frente a los problemas de liquidez y solvencia que se encuentre pasando una entidad financiera. Por el lado del sector financiero popular y solidario se cuenta con un fondo de USD 1.170.436.415,24. Estos valores son enviados por las entidades financieras a la COSEDE para prevenir cualquier inconveniente que ponga en riesgo los ahorros de los usuarios. Con la aplicación de las encuestas a la población ecuatoriana se conoció que aproximadamente el $91 \%$ de los usuarios desconocen sobre la institución, sus gestiones y procedimientos. Los resultados que seobtuvieron fueron: alrededor del $96 \%$ de los usuarios poseen una cuenta de ahorro; el $91 \%$ de la muestra desconoce acerca de las gestiones y procedimientos de la COSEDE, mientras que el $89 \%$ carece de información sobre el seguro de depósito, es decir hay ausencia de cultura financiera en la población.

Estos resultados indican que la población desconoce que sus ahorros se encuentran protegidos por la COSEDE y que ante cualquier problema de liquidación forzosa que presente una determinada entidad bancaria, la institución resguardará los depósitos de todos los clientes, llegando a cancelar el valor máximo de cobertura de los ahorros, esto se lograría a través del fondo que las instituciones financieras destinan a la COSEDE como un mecanismo de contingencia financiera. Además, dentrodel programa COSEDE territorial, se pudo comprobar que en 2018 de las 24 provincias del país solamente 15 de ellas han tenido capacitaciones sobre educación financiera, donde sumó un total de 2.416 personas instruidas, quedando pendiente 9 provincias por capacitar.

\section{Conclusiones}

La creación de la Corporación del Seguro de Depósitos, Fondo de Liquidez y Fondo de Seguros Privados (COSEDE) se aprobó en el año 2008 con la Ley de Creación de la Red de Seguridad Financiera. En el período de estudio 2013 - 2018, se observó el crecimiento de los tres fondos que resguarda la institución: seguro de depósito, fondo de liquidez y fondo de seguros privados. Estos actúan como un mecanismo de contingencia financiera para cuando se presente una liquidación en una deteminada entidad financiera.

El seguro de depósito consolidado registra un valor de USD 8.111.271.947,37 monto que permitirá a la institución actuar de manera inmediata y eficaz ante cualquiera liquidación de las entidades financieras privadas y de la Economía Popilar y Solidaria, esto con el objetivo de resguardar el dinero de los ciudadanos. Por el lado del fondo de liquidez se presentó un valor total de USD 13.881.236.242,37 este permitirá la otorgación de préstamos para aquellas entidades que presenten problema de liquidez y solvencia. Y el fondo de seguros privados, a pesar que 
registra información desde el 2016, también ha tenido un crecimiento permitiendo así hacer frente a liquidaciones forzosas que sucedan en el Sistema de seguros privados

Mediante las herramientas metodológicas se verificó que la Corporación del Seguro de Depósitos, Fondo de Liquidez y Fondos de Seguros Privado (COSEDE), en el período 2013-2018, ha ayudado a la ciudadanía a recuperar sus ahorros cuando las instituciones financieras han atravesado por liquidaciones forzosas, según la ley COMF. Además, se consiguió evidenciar que la mayoría de la población ecuatoriana desconoce los procedimientos y gestiones que realiza esta institución, esto se observó directamente en las encuestas aplicadas en el país.

\section{Referencias bibliográficas}

Apolinario Quintana, R., Valle, J., \& Caicedo , M. (Julio de 2017). “Evolución de los seguros de depósitos en las cooperativas de ahorro y crédito en el Ecuador y su importancia en la estabilidad financiera nacional. Observatorio de la Economía Latinoamericana. Recuperado el 11 de Junio de 2019, de http://www.eumed.net/cursecon/ecolat/ec/2017/cooperativas-credito-ecuador.html

Asociación Internacional de Aseguradores de Depósitos. (s.f.). ¿Qué es la IADI? Recuperado el 20 de Julio de 2019, de https://www.iadi.org/en/

Corporación de Seguro de Depósitos, Fondo de Liquidez y Fondo de Seguros Privados. (Septiembre de 2013). Las redes de seguridad financiera y los seguros de depósitos: importancia y rol para la estabilidad financiera. Recuperado el 6 de Julio de 2019, de Corporación del Seguro de Depósitos, Fondo de Liquidez y Fondo de Seguros Privados (COSEDE): http://www.cosede.gob.ec/wpcontent/uploads/2013/09/presentacionmiguelruiz.pdf

Corporación de Seguros de Depósitos, Fondo de Liquidez y Fondo de Seguros Privados. (s.f.). Misión. Recuperado el 10 de Julio de 2019, de COSEDE: https://www.cosede.gob.ec/vision-mision-valores/

Corporación del Seguro de Depósitos Fondo de Liquidez y Fondo de Seguros Privados. (2014). Informe preliminar de rendición de cuentas. Recuperado el 8 de Junio de 2019, de http://www.cosede.gob.ec/wpcontent/uploads/2015/04/Rendicion-de-cuentas-final-2014.pdf

Corporación del Seguro de Dépositos, Fondo de Liquidez y Fondo de Seguros Privados. (s.f.). Fondo de liquidez. Recuperado el 30 de Julio de 2019, de COSEDE: https://www.cosede.gob.ec/fondo-de-liquidez/

Corporación del Seguro de Dépositos, Fondo de Liquidez y Fondo de Seguros Privados. (s.f.). Fondo de Seguros Privados. Recuperado el 30 de Julio de 2019, de COSEDE: https://www.cosede.gob.ec/fondo-de-segurosprivados/

Corporación del Seguro de Depósitos, Fondo de Liquidez y Fondos de Seguros Privados. (s.f.). Visión. Recuperado el 10 de Julio de 2019, de COSEDE: https://www.cosede.gob.ec/vision-mision-valores/

Corporación del Seguros de Depósitos, Fondo de Liquidez y Fondo de Seguros Privados. (Julio de 2018). Plan estratégico institucional 2017 - 2021. Recuperado el 10 de Julio de 2019, de Corporación del Seguros de Depósitos, Fondo de Liquidez y Fondo de Seguros Privados: http://www.cosede.gob.ec/wpcontent/uploads/2018/07/Plan\%20Estrat\%C3\%A9gico\%20Institucional\%20\%20COSEDE\%202017-2021.pdf

Garabiza Castro, B. (Septiembre de 2014). El rol de la red de seguridad financiera, los seguros de depósitos y su importancia para la estabilidad financiera ecuatoriana: periodo 2007-2013. Recuperado el 11 de Junio de 2019, de Universidad de Guayaquil:

http://repositorio.ug.edu.ec/bitstream/redug/6275/1/TESIS\%20BELLA\%20GARABIZA\%20CASTRO\%20\%20f inal.pdf 
Gómez Bastar, S. (2012). Metodología de la investigación (Primera ed.). Tlalnepantla: Red Tercer Milenio S.C. Obtenido de http://www.aliat.org.mx/BibliotecasDigitales/Axiologicas/Metodologia_de_la_investigacion.pdf

Guerrero, R., Focke, K., \& Rossini, F. (2010). Redes de Seguridad Financiera. Aspectos Conceptuales y Experiencias Recientes en América Latina y el Caribe. Universidad de las Américas, Centro de Investigaciones Económicas, Quito. Recuperado el 19 de Junio de 2019, de https://www.udla.edu.ec/cie/wp-content/uploads/2016/09/Working-Paper-N\%C2\%B05-Redes-deSeguridad-Financiera-R-Guerrero-K-Focke-y-F-Rossini.pdf

Hernández Sampieri, R., Fernández Collado, C., \& Baptista Lucio, M. (2014). Metodología de la investigación (6ta ed.). México D.F, México: McGraw-Hill. Recuperado el 2 de Febrero de 2020, de https://www.uca.ac.cr/wp-content/uploads/2017/10/Investigacion.pdf

Rivas García, M. V. (Mayo de 2016). Implicaciones de los acuerdos de Basilea III para el sistema bancario salvadoreño. Recuperado el 16 de Julio de 2019, de Universidad de El Salvador: http://ri.ues.edu.sv/id/eprint/14959/1/Implicaciones\%20de\%20los\%20Acuerdos\%20de\%20Basilea\%20par a\%20el\%20Sistema\%20Bancario\%20Salvadore\%C3\%B1o.pdf.

Esta obra está bajo una Licencia Creative Commons

Attribución-NoCommercial 4.0 International

(cc) EY-NC 